\title{
AN EXTENSION OF AN OPERATOR INEQUALITY FOR $s$-NUMBERS
}

\author{
JAMES A. COCHRAN
}

\begin{abstract}
If it is assumed that the s-numbers associated with a given compact operator are ordered in the usual fashion, a basic result concerning infinite series of powers of these $s$-numbers can be appropriately restated so as to refer solely either to the lead terms of the series or to its tail. A simple proof, based upon an interesting auxiliary result concerning stochastic matrices, is given for this useful improvement.
\end{abstract}

A fundamental result in operator theory (see, for example, McCarthy [6], Gohberg and Krein [5], or Dunford and Schwartz [3]) relates infinite power sums of the $s$-numbers (characteristic numbers) of a given operator and corresponding power sums of the norms of the images, under the operator, of orthonormal basis vectors for the underlying Hilbert space. In the case of a compact operator $T$ acting on a Hilbert space $\mathcal{H}$, the following represents an extension of this result found useful in recent investigations of integral operators [2], [7]:

THEOREM. If $0<r \leqslant 2$, then for arbitrary $m \geqslant 1$,

$$
\sum_{n=m}^{\infty}\left[s_{n}(T)\right]^{r}=\inf \sum_{n=m}^{\infty}\left\|T \Psi_{n}\right\|^{r},
$$

while if $2 \leqslant r<\infty$,

$$
\sum_{n=1}^{m}\left[s_{n}(T)\right]^{r}=\sup \sum_{n=1}^{m}\left\|T \Psi_{n}\right\|^{r} \text {. }
$$

In these expressions the $s$-numbers are ordered in their natural nonincreasing manner and the inf and sup are taken over all orthonormal bases of $\mathcal{H}$. If $r \neq 2$, equality occurs, as expected, if and only if $\left\{\Psi_{n}\right\}$ is an appropriately ordered orthonormal basis for $\mathcal{H}$ consisting of characteristic functions (eigenvectors) for the related nonnegative definite operator $T^{*} T$.

The proof of (2) can be accomplished by employing several auxiliary results of Gohberg and Kreìn [5]. Alternatively, both (1) and (2) can be established making appropriate use of a theorem of Fan [4] (see also Beckenbach and Bellman [1, p. 77]). A simple and unencumbered demonstration, however, can be based directly upon the following result on doubly-stochastic matrices-a

Received by the editors May 11, 1976 and, in revised form, June 28, 1976.

AMS (MOS) subject classifications (1970). Primary 47B05, 47A10; Secondary 47B10. 
result which was subsidiary to the Fan theorem, but certainly is of interest in its own right.

LEMMA. Let the nonnegative elements $p_{i j}(i, j=1,2, \ldots)$ be such that

$$
\sum_{i=1}^{\infty} p_{i j} \leqslant 1\left(j=1,2, \ldots, \quad \sum_{j=1}^{\infty} p_{i j} \leqslant 1(i=1,2, \ldots,)\right. \text {. }
$$

If $a_{i}, b_{i}(i=1,2, \ldots)$ are two nonincreasing sequences of nonnegative numbers, then

$$
\sum_{i=1}^{m} a_{i} \sum_{j=1}^{n} p_{i j} b_{j} \leqslant \sum_{i=1}^{m} a_{i} b_{i}
$$

In order to prove the main theorem, we let $\left\{\Phi_{n}\right\}$ be a sequence of orthonormalized characteristic functions of the operator $T^{*} T$ corresponding to the naturally ordered $s$-numbers $s_{n}^{2}(T)$. Then for arbitrary $\Psi$ in $\mathcal{H}$ and $r \geqslant 2$,

$$
\begin{aligned}
\|T \Psi\|^{r} & =\left\{\sum_{j=1}^{\infty}\left[s_{j}(T)\right]^{2}\left|\left(\Psi, \Phi_{j}\right)\right|^{2}\right\}^{r / 2} \\
& \leqslant \sum_{j=1}^{\infty}\left[s_{j}(T)\right]^{r}\left|\left(\Psi, \Phi_{j}\right)\right|^{2}\left\{\sum_{j=1}^{\infty}\left|\left(\Psi, \Phi_{j}\right)\right|^{2}\right\}^{(r-2) / 2},
\end{aligned}
$$

by Hölder's inequality. If $\left\{\Psi_{n}\right\}$ is an orthonormal basis for $\mathcal{H}$, it follows, using Parseval's relation, that

$$
\sum_{n=1}^{m}\left\|T \Psi_{n}\right\|^{r} \leqslant \sum_{n=1}^{m} \sum_{j=1}^{\infty}\left[s_{j}(T)\right]^{r}\left|\left(\Psi_{n}, \Phi_{j}\right)\right|^{2} .
$$

This inequality and the identifications $a_{i}=1, b_{j}=\left[s_{j}(T)\right]^{r}$, and $p_{i j}=$ $\left|\left(\Psi_{i}, \Phi_{j}\right)\right|^{2}$ in the Fan Lemma immediately lead to the desired result, namely

$$
\sum_{n=1}^{m}\left\|T \Psi_{n}\right\|^{r} \leqslant \sum_{n=1}^{m}\left[s_{n}(T)\right]^{r}
$$

In the case of $0<r \leqslant 2$, the comparable chain of inequalitities is

$$
\begin{aligned}
\sum_{n=m}^{\infty}\left\|T \Psi_{n}\right\|^{r} & \geqslant \sum_{n=m}^{\infty} \sum_{j=1}^{\infty}\left[s_{j}(T)\right]^{r}\left|\left(\Psi_{n}, \Phi_{j}\right)\right|^{2} \\
& =\sum_{j=1}^{\infty}\left[s_{j}(T)\right]^{r}\left[1-\sum_{n=1}^{m-1}\left|\left(\Psi_{n}, \Phi_{j}\right)\right|^{2}\right] \\
& \geqslant \sum_{n=m}^{\infty}\left[s_{n}(T)\right]^{r} .
\end{aligned}
$$

When $\Psi_{n}=\Phi_{n}(n=1,2, \ldots)$, all the various inequalities above become equalities-and when $r \neq 2$, only then-thereby completing the proof of (1), (2) and the Theorem. 


\section{REFERENCES}

1. E. F. Beckenbach and R. Bellman, Inequalities, Springer-Verlag, New York, 1965. MR 33 \#236.

2. J. A. Cochran, Composite integral operators and nuclearity, Ark. Mat. (to appear).

3. N. Dunford and J. T. Schwartz, Linear operators. Part II, Interscience, New York, 1963. MR 32 \#6181.

4. K. Fan, Maximum properties and inequalities for the eigenvalues of completely continuous operators, Proc. Nat. Acad. Sci. U.S.A. 37 (1951), 760-766. MR 13, 661.

5. I. C. Gohberg and M. G. Krein, Introduction to the theory of linear nonselfadjoint operators, "Nauka", Moscow, 1965; English transl., Transl. Math. Monographs, vol. 18, Amer. Math. Soc., Providence, R.I., 1969. MR 36 \# 3137; 39 \# 7447.

6. C. A. McCarthy, $c_{p}$, Israel J. Math. 5 (1967), 249-271. MR 37 \# 735.

7. C. Oehring and J. A. Cochran, Integral operators and an analogue of the Housdorff-Young theorem, J. London Math. Soc. (to appear).

Department of Mathematics, Virginia Polytechnic Institute and State University, Blacksburg, Virginia 24061 